\title{
ANÁLISIS JURÍDICO DE LA SERIE “13 REASONS WHY"*
}

\author{
LEGAL ANALYSIS OF THE TV SERIES "13 REASONS WHY”
}

\author{
Vanessa Antuanet Abreu Sancho ${ }^{1}$
}

Elizabeth Huamán Cruz²

\section{Resumen}

El presente artículo aborda diversos problemas jurídicos que surgen en torno a la serie televisiva "13 Reasons Why", entre los que se encuentran la inducción al suicidio, la violación sexual, el bullying, entre otros; los que son analizados no solo desde una perspectiva jurídica, sino también bioética. Para finalmente, determinar si estos actos constituyen delito y si cabe interponer algún tipo de acción penal en contra de quienes los cometieron.

Palabras clave: Inducción al suicidio, Violación sexual, Responsabilidad, Violencia escolar

\begin{abstract}
:
This article studies the legal problems that arise around the series "13 Reasons Why" among which are the induction to suicide, rape, bullying, among others, these are analyzed not only from a legal perspective but also bioethics. Finally, to determine if these acts constitute a crime and if it is possible to file some kind of criminal action against those who committed them.
\end{abstract}

Keywords: Induction to suicide, Rape, Responsibility, School violence.

El presente artículo constituye una versión corregida del trabajo que sobre la materia prepararon las autoras como parte del curso: Seminario de Bioética y Biojurídica, dictado en el semestre 2018 - I, en la Facultad de Derecho de la UNIFE por el Mag. Ronald Cárdenas Krenz.

1 Alumna del X ciclo de la Facultad de Derecho de la UNIFE, ex Representante ante el Consejo Estudiantil de la Facultad de Derecho de la UNIFE.

2 Alumna del X ciclo de la Facultad de Derecho de la UNIFE, ex Representante ante el Consejo Estudiantil de la Facultad de Derecho de la UNIFE. 


\section{INTRODUCCIÓN}

El suicidio juvenil es un problema que afecta seriamente a la población, siendo grave especialmente en los jóvenes. Conforme señalan Bedoya \& Montaño (2016), citando a La Organización Mundial de la Salud (OMS), el suicidio es "el acto deliberado de quitarse la vida". Desde 1970 es considerado como un problema de salud pública. Según el Instituto Nacional de Salud Mental de Lima, se presentan de 1 a 3 suicidios al día en nuestro país y alrededor de un $8.9 \%$ de peruanos ha pensado en quitarse la vida en algún momento.

En el año 2017, se estrenó "13 Reasons Why", basada en la novela escrita por Jay Asher en 2008, convirtiéndose en una de las series más populares de Netflix en diversos países, especialmente entre los adolescentes.

El argumento gira en torno a los hechos que llevaron a una adolescente ficticia, llamada Hannah Baker, de 17 años, a tomar la drástica decisión de acabar con su vida, dejando testimonio de las razones que la llevaron a ello a través de 13 cassettes ${ }^{13}$. Fallecida la menor, sus padres inician una investigación con el fin de saber qué sucedió con su hija, encontrar responsables e iniciar las acciones legales correspondientes.

\section{ANTECEDENTES}

Durante los 13 episodios que tiene la primera temporada, se observa cómo la protagonista vive distintas situaciones que la involucran a ella y a diversos compañeros de su escuela.

Todo lo vivido y descrito por Hannah Baker, que de por sí son situaciones traumáticas para cualquier persona adulta, lo son más para una adolescente que no cuenta con un soporte emocional que la ayude a superar las circunstancias difíciles de las que es víctima; sus padres están distantes debido a la mala situación económica que atraviesan; y, además, las personas a las que ella considera sus mejores amigos, se alejan para ir en busca de nuevas amistades e intereses.

\section{ANÁLISIS JURÍDICO}

Esta serie tiene relevancia jurídica porque desarrolla a lo largo de sus 13 capítulos distintos temas como:

Ante el éxito de la serie, Netflix decidió grabar otra temporada, la que fue lanzada en mayo del 2018. Se calcula que ha sido vista por 6,08 millones de espectadores en sus 3 primeros días de estreno. Los personajes principales de la serie son: Clay Jensen, Hannah Baker, Bryce Walker, Jessica Davis, Justin Foley y Tony Padilla. 


\section{- La búsqueda de un responsable del suicidio de Hannah Baker.}

Respecto a este punto, es necesario analizar si corresponde imputar la responsabilidad de esta conducta a alguno de los compañeros de escuela de la protagonista; para ello, consideramos necesario hacer referencia al delito de "Instigación o ayuda al suicidio" de nuestro Código Penal. Del análisis de esta figura, se puede advertir que este es un delito DOLOSO, por lo que es necesario que el sujeto activo busque con su accionar que el sujeto pasivo, en este caso Hannah Baker, acabe con su vida.

Sin embargo, en la serie no se evidencia que quienes abusaron y vulneraron los derechos de la protagonista, por más viles y perversos que sean, buscasen que ella acabe con su vida; es decir, ellos no tenían la intención de que ella se suicide. Es más, en el primer capítulo de la primera temporada, la propia Hannah señala que quienes ella señala como responsables de su muerte no sabían lo que sus acciones iban a desencadenar.

Por lo tanto, lo ocurrido con Hannah Baker, no reúne los presupuestos necesarios para ser considerado como "Instigación o ayuda al suicidio", consecuentemente, no existe responsabilidad penal imputable a alguno de sus compañeros respecto a esta figura delictiva.

\section{- El abuso sexual.}

La violación sexual ${ }^{5}$ se encuentra tipificada en el artículo $170^{\circ}$ de nuestro Código Penal, este tema se aborda en más de un capítulo de la serie, ya que la protagonista y una de sus compañeras de escuela, el personaje Jessica Davis, fueron abusadas sexualmente. Sin embargo, ninguna de ellas denunció los hechos, y estos se hicieron públicos recién mediante los audios dejados por Hannah Baker tras su muerte.

Es necesario precisar, que en el caso de Hannah Baker, se torna difícil la obtención de alguna prueba que corrobore que este hecho sucedió, debido a que solo se cuenta con la declaración de la víctima en un casete, quien no realizó la denuncia correspondiente $y$, además, no es posible que se puedan realizar los exámenes médicos necesarios para acreditar los hechos.

\footnotetext{
4 Artículo $113^{\circ}$.- Instigación o ayuda al suicidio

“El que instiga a otro al suicidio o lo ayuda a cometerlo, será reprimido, si el suicidio se ha consumado o intentado, con pena privativa de libertad no menor de uno ni mayor de cuatro años.

La pena será no menor de dos ni mayor de cinco años, si el agente actuó por un móvil egoísta."

5 Artículo $170^{\circ}$.- Violación sexual

"El que con violencia o grave amenaza, obliga a una persona a tener acceso carnal por vía vaginal, anal o bucal o realiza otros actos análogos introduciendo objetos o partes del cuerpo por alguna de las dos primeras vías, será reprimido con pena privativa de libertad no menor de seis ni mayor de ocho años." (...)
} 
Conforme señalan García Matamoros \& Ávila Medina (2017, p.174) al referirse a la violación sexual, “(...) dada la naturaleza de esta forma de violencia, no se puede esperar la existencia de pruebas gráficas o documentales y, por ello, la declaración de la víctima constituye una prueba fundamental sobre el hecho." Sin embargo, en el presente caso no existe una declaración formal de la víctima y tampoco sería posible obtener una, debido a que ella falleció.

Por otro lado, también se debe analizar la declaración del violador, obtenida por un compañero de escuela (Clay Jensen), sobre el particular, esta declaración tampoco podría ser utilizada como prueba, toda vez que fue obtenida mediante el uso de la violencia; además, quien confesó podría alegar que lo dijo con la finalidad de generar confusión o para evitar que continúe la agresión hacia él.

Respecto a la violación sexual de Jessica Davis, sí se podría probar la existencia de este delito, debido a que la víctima puede ser sometida a los exámenes médicos pertinentes y ofrecer su declaración con el fin de iniciar un proceso penal en contra de su agresor.

Asimismo, es necesario referirnos a la persona que comete estos delitos: Bryce Walker, ya que si bien es cierto no puede ser procesado por la violación de Hannah Baker, sí puede serlo por la de Jessica Davis, solo es necesario que ella decida denunciarlo.

\section{- La violencia en el ámbito escolar.}

Este es un problema recurrente en nuestra época; resulta curioso cómo es que los personajes de la serie, adolescentes, tienen gran facilidad para conseguir armas de fuego, situación que no dista de la realidad debido a que se han registrado numerosos tiroteos en las escuelas de Estados Unidos en los últimos años. Muestra de ello es uno de los últimos tiroteos ocurrido en una escuela de EEUU originado por un estudiante en el Distrito Escolar de Santa Fe, Texas, quien provocó la muerte de al menos 10 personas y dejó varios heridos; en el presente año, se han registrado 22 tiroteos en centros educativos de los Estados Unidos. (El Tiempo, 2018)

Si bien es cierto, en nuestro país no se dan situaciones similares, la violencia en el ámbito escolar no escapa a nuestra realidad, prueba de ello es el reciente caso ocurrido en Satipo (Junín), donde un estudiante del cuarto grado de primaria, quien había sido expulsado de su colegio por mala conducta, incendió su centro de estudios (RPP, 2018), si bien este hecho, felizmente, no comprometió 
la integridad física de sus compañeros y personal administrativo, generó la destrucción de los materiales didácticos y mobiliarios de la escuela; suceso que evidencia el grado de violencia presente en nuestros centros educativos.

\section{CASO PERUANO}

Distintos medios de comunicación de nuestro país informaron el caso de un joven ingeniero arequipeño de 23 años, Franco Alonso Lazo Medrano ${ }^{6}$, quien, según refieren, se suicidó siguiendo la dinámica de la serie; es decir, dejó unos audios e instrucciones para las personas a las que iban dirigidas las grabaciones que se encontraban en su computadora. Sin embargo, si bien se dejaron audios para distintas personas, no se puede afirmar que él haya tomado la decisión de acabar con su vida para imitar el accionar de la protagonista de la serie, ya que una persona no decide poner fin a su vida por el solo hecho de imitar algún comportamiento, sino que deben haber ocurrido situaciones en su vida personal que lo llevaran a suicidarse.

\section{NUESTRA POSICIÓN}

Después de haber analizado la serie a grandes rasgos, consideramos necesario realizar las siguientes preguntas:

\section{1. ¿El suicidio de Hannah Baker representa un delito?}

Si bien nuestra posición ya quedó clara con lo explicado líneas arriba, es necesario recalcar que el suicidio de Hannah Baker por más terrible y triste que sea, no representa delito, no nos encontramos ante un caso de "Instigación o ayuda al suicidio", en tanto no se cumple con el requisito establecido por nuestro ordenamiento jurídico, que es, la existencia de dolo por parte del (o de los) sujeto (s) activo(s) del delito, no hay intención de que la protagonista termine con su vida, ya que como señala Bramont-Arias (1994, p.58), la acción consiste en instigar, es decir, "provocar incitar, o inducir a otro a que se suicide. Esta instigación debe tender a que el inducido ponga fin a su vida y debe dirigirse a persona determinada".

De lo anterior, se puede deducir que para que se configure el delito de "Instigación o ayuda al suicidio", las sugerencias que se dan a la persona para que ponga fin a su vida, deben ser categóricas, es decir, no basta con el solo hecho de hacer insinuaciones; tampoco el hecho de que la protagonista de

La Policía encontró dentro de su cuarto dos notas suicidas, una donde daba instrucciones y nombres de las personas para que escuchen unos audios que dejó en su computadora. Obtenido de: https://larepublica.pe/sociedad/882870-arequipa-joven-se-suicida-por-decepcion-amorosa-y-deja-indicaciones-para-escuchar-audios 
la serie haya sufrido maltratos físicos y psicológicos puede ser considerado como instigación. Fue ella quien tomó la decisión de acabar con su vida para evadir las situaciones violentas de las que era víctima, sin que ninguno de sus compañeros le haya sugerido que recurra al suicidio. En todo caso, se puede señalar que las personas de su entorno actuaron de manera indiferente ante los problemas que la protagonista enfrentaba.

\section{2. ¿La serie incentiva de alguna manera el suicidio en los adolescentes?}

Consideramos que el contenido de la serie si bien muestra situaciones difíciles para muchos de los espectadores, evidencia circunstancias que lamentablemente se dan a diario y que muchas veces desconocemos, ya que quienes sufren de abuso no lo manifiestan o simplemente no se perciben las señales que ellos transmiten.

Concordamos con Horrillo (2017) cuando señala que esta "no es una serie sobre el bullying, el suicidio, el machismo, las violaciones o las redes sociales. Es sobre cómo los adolescentes del siglo XXI se enfrentan a todos esos problemas y cómo los adultos son incapaces de verlo".

Cuando se estrenó la serie, generó gran controversia debido a que muchos especialistas en salud mental de Estados Unidos estimaron que "13 Reasons Why" podría generar un "efecto contagio"7 en la audiencia." (Diario La República, 2018).

Se ha dicho, por ejemplo, que la serie “(...) no proporcionó suficientes referencias respecto a los recursos de prevención del suicidio para personas que puedan estar en riesgo." (Diario El Comercio, 2018). También se le ha acusado "(...) de mostrar una visión polémica y edulcorada del suicidio." (Diario El Comercio, 2018).

También se han publicado noticias sobre la serie, como sigue:

"Una madre de Florida ha acusado a '13 Reasons Why' del intento de suicidio de su hija, una adolescente de 15 años que trató de quitarse la vida el pasado Día de la Madre" (Diario El Comercio, 2018).

“Escuelas primarias de Canadá emitieron un comunicado enviado a los padres advirtiendo el contenido de la serie: acusa de hacer una "glamourización del comportamiento suicida" (Del Moral, 2017)

También conocido como "Efecto Werther", debido a que en el siglo XVIII, cuando la novela "Las penas del joven Werther" fue prohibida porque, una serie de suicidios similares a los del libro. 
Sin embargo, según "una investigación realizada por la Universidad de Northwestern, 13 Reasons Why, funcionó como una herramienta educativa para los jóvenes, fomentó las charlas entre padres e hijos, e inspiró más compasión entre los adolescentes." (Karter, 2018).

Entonces, para contestar la pregunta de si la serie busca incentivar el suicidio en los adolescentes, nuestra respuesta es NO, porque así como algunas personas podrían pensar que quienes ven esta serie buscarán imitar el comportamiento de Hannah Baker para acabar con sus problemas, ¿por qué no podemos creer que tendrá un efecto contrario?; es decir, que desincentivará a los jóvenes a que acaben con su vida, que entiendan que esa no es la salida a sus problemas, sino que por el contrario, es el comienzo del caos en las vidas de todos aquellos a quienes dejan atrás.

Adicionalmente, es importante que aquellos cercanos al entorno de los adolescentes, como padres o familiares que viven con ellos, así como profesores o consejeros en los colegios, deben estar pendientes si existen signos de alerta de que estos jóvenes poco a poco se están desligando de todos, ya que al desligarse dejan de tener un soporte, que los hace vulnerables y los predispone a tomar este tipo de decisiones.

\section{CONCLUSIONES}

Durante la serie, observamos que la protagonista fue víctima de abusos y agresiones de diferente tipo, lo que la llevó a tomar la decisión equivocada de acabar con su vida; sin embargo, la responsabilidad de este hecho no puede ser imputada a una persona distinta a la misma Hannah Baker, debido a que no concurrieron los elementos necesarios para la configuración del delito de "Instigación o ayuda al suicidio".

Por otro lado, que esta serie que aborda situaciones violentas sea vista por adolescentes o menores de edad no representa un peligro para ellos, ya que si los temas tratados en la serie son conversados con algún adulto responsable podrán ser entendidos por aquellos más vulnerables que sientan que están en la misma situación que Hannah. 13 Reasons Why debe actuar como un disuasorio para que los jóvenes no sientan que el suicidio es la solución para sus problemas.

No obstante, en el caso de la violación sexual sufrido por la protagonista, sí existe delito debido a que el hecho fue consumado; sin embargo, el problema se centra en si existen pruebas suficientes que permitan iniciar una acción contra el agresor, debido a que Hannah Baker falleció sin haber 
efectuado una denuncia sobre el abuso, solo dejó una cinta en la que narra los hechos. Por otro lado, la violación sexual sufrida por Jessica Davis, sí podría ser llevada a juicio, si es que ella decide denunciar los hechos.

\section{REFERENCIAS}

Bakalarz, B., \& Pappolla, R. (s.f.). Suicidio en adolescentes. Recuperado de: http://www.sap.org.ar/docs/SUICIDIO.pdf

Barón, O. (agosto de 2000). Adolescencia y suicidio. Psicología desde el Caribe, 48-69. Recuperado de : http:/ / www.redalyc.org/pdf/213/21300605.pdf

Bedoya,E., \& Montaño, L. (19 deJulio de2016). Suicidio y trastornomental. Recuperado de : http://www.redalyc.org/jatsRepo/4235/423548400012/ index.html

Bramont-Arias, L. (1994). Manual de derecho penal: Parte especial. Lima: San Marcos.

Del Moral, M. (06 de mayo de 2017). La polémica alrededor de "13 Reasons Why": ¿concientización o incitación? Recuperado de : https://www.infobae. com/tendencias / 2017/05/06/la-polemica-alrededor-de-13-reasons-whyconcientizacion-o-incitacion/

Diario El Comercio (20 de mayo de 2018). 13 Reasons Why confronta las críticas por su contenido con estas advertencias. Recuperado de : https:/ / elcomercio.pe/tvmas/series/netflix-13-reasons-why-advertenciaspublico-contenido-noticia-521151

Diario El Comercio (15 de mayo de 2018). Acompañamiento de un adulto es importante para los adolescente que miran 13 Reasons Why. Recuperado de: http: / / www.elcomercio.com/tendencias/acompanamiento-adulto-adolescentesserie-13reasonswhy.html

Diario La República (18 de mayo de 2018). Resumen en 2 minutos de la primera temporada de 13 Reasons Why. Recuperado de: https:/ / larepublica. pe/cine-series/1244243-13-reasons-why-2-resumen-primera-segundatemporada-serie-netflix

El Tiempo (18 de mayo de 2018). Diez muertos en tiroteo en escuela de Texas, EE. UU. Recuperado de : http:/ / www.eltiempo.com/mundo/eeuu-ycanada/tiroteo-en-escuela-de-santa-fe-texas-estados-unidos-219070 
García-Matamoros, L., \& Ávila-Medina, D. (2017). Procedimiento, litigio y representación ante tribunales internacionales. Bogotá: Universidad del Rosario.

Guía Salud (s.f.). Guía de Práctica Clínica sobre la Depresión Mayor en la Infancia y en la Adolescencia. Recuperado de http://www.guiasalud.es/egpc/ depresion_infancia/completa/documentos/apartado09/apartado09.pdf

Horrillo, E. (05 de abril de 2017). Por qué padres y adolescentes deben ver la cruda nueva serie de Selena Gómez. Obtenido de El país: https: / / elpais.com/ elpais/2017/04/04/icon/1491299079_468707.html

Karter, E. (21 de marzo de 2018). Estudio multinacional: Cómo reaccionaron los adolescentes y sus padres al ver la serie de Netflix " 13 Reasons Why". Recuperado de: https://13reasonsresearch.soc.northwestern.edu/ netflix_latin-american-release.pdf

Moya, J. (2007). La conducta suicida en adolescentes, sus implicaciones en el ámbito de la justicia juvenil. Recuperado de : http:/ / www.osamcat.cat/cat/ Publicacions/Articles/ConductaSuicida.pdf

Pacheco, B., \& Peralta, P. (2015). La conducta suicida en la adolescencia y sus condiciones de riesgo. ARS Médica: Revista de ciencias médicas, 40(1), 47-55. Recuperado de: http://www.arsmedica.cl/index.php/MED/article/view/38

RPP. (22 de mayo de 2018). Menor de edad es investigado por ocasionar un incendio en su escuela en Satipo. Recuperado de : http:/ / rpp.pe/peru/junin/ menor-de-edad-es-investigado-por-ocasionar-un-incendio-en-su-escuelaen-satipo-noticia-1124256

Vargas,H.,\&Saavedra,J. (2012).Factoresasociadosconlaconductasuicida enadolescentes. Revistadeneuropsiquiatría,75(1),19-28. Recuperadode: http:/ / repositorio.minedu.gob.pe/bitstream/handle/123456789/1294/2012 Vargas_Factores $\% 20$ asociados\%20con $\% 201$ a $\% 20$ conducta $\% 20$ suicida $\% 20$ en $\% 20$ adolescentes.pdf? sequence $=1 \&$ isAllowed $=y$

Fecha de recepción $\quad$ : 27 de agosto de 2018

Fecha de aceptación : $\quad 28$ de octubre 2018 